\title{
Hypertension and Its Treatment in the Elderly
}

\author{
Matteo Rigato, Laura Gobbi, Francesca Simioni, Luciana Bonfante, Federico Nalesso and Lorenzo A Calò* \\ Department of Medicine, Nephrology, Dialysis and Transplantation Unit, Italy
}

Received: 阱 December 24, 2018; Published: 制 January 16, 2019

*Corresponding author: Lorenzo A Calò, MR and LG, Department of Medicine (DIMED), Nephrology, Dialysis and Transplantation Unit, Italy, Email: renzcalo@unipd.it, MR and LG equally contributed to this work.

\section{Mini Review}

The treatment of hypertension has been shown to be accompanied by a reduction in mortality and morbidity both in older and younger hypertensive patients. In the elderly hypertensive patients BP should be lowered to a systolic value of $130-139 \mathrm{mmHg}$ and a diastolic value of $<80 \mathrm{mmHg}$ if tolerated. Treated SBP values of $<130 \mathrm{mmHg}$ should be avoided and in the elderly hypertensive patients it should be advised to undertake treatment together with an evaluation of the presence of other comorbidities and the risks associated with multi-therapy, especially in the older. Hypertension is a known risk factor for cardiovascular morbidity and mortality and in the long term one of the main causes of end stage renal disease. The definition of hypertension does not change with age: office blood pressure value at least $140 \mathrm{mmHg}$ for systolic blood pressure (SBP) and diastolic blood pressure (DBP) at least 90 $\mathrm{mmHg}$ is defined as grade 1 hypertension [1].

Although rise in blood pressure is not a normal part of aging, the incidence of hypertension in the elderly population is high. In the 1988-2004 National Health and Nutrition Examination Survey $67 \%$ of adults aged 60 and older were found to be hypertensive [2]. Data from the Framingham study show that $58.9 \%$ of people aged $\geq 65$ and $70 \%$ of those aged $\geq 80$ years are hypertensive [3]. SBP rises progressively until the age of 70 or 80 , whereas DBP increases until the age of 50 or 60 and then tends to level or even decline slightly. This combination of changes likely reflects aged related increase in stiffness of the blood vessels [4]. Arterial compliance is reduced by wall thickening, calcium accumulation, quantitative and qualitative alterations in collagen [5]. Baroreceptors sensitivity is altered with age and this may explain the variability of blood pressure revealed by continuous monitoring [6]. Vascular oxidative stress increases with aging, leading to pro-inflammatory phenotypical changes and an altered endogenous bioavailability of vasoactive substances with consequent impaired vascular function and changes in smooth muscle tone; this leads to an impairment of postural reflexes, making elderly hypertensive individuals more sensitive to orthostatic hypotension [7].

The loss of diastolic augmentation caused by thestiffness ofmajor arteries leads to a fall in perfusion pressure in the coronary arteries, and changes in cerebrovascular circulation lead to reductions in cerebral perfusion [8]. Renal perfusion decreases with aging and determine an impairment of renal function with and reduction of the glomerular filtration rate [9]. All these changes could explain the decreased cardiac output, decreased heart rate, decreased myocardial contractility, nephroangiosclerosis, left ventricular hypertrophy seen in elderly patients and increased cardiovascular risk respect to general population. The cardiocerebrovascular-renal risk from hypertension is demonstrated by the higher incidence of stroke, left ventricular hypertrophy, congestive heart failure, coronary and peripheral artery diseases, vision impairment, endstage renal disease, cognitive impairment, and dementia seen in the elderly hypertensive patients [10]. A large amount of evidences demonstrates that lowering blood pressure can substantially reduce this high morbidity and mortality [11-13].

"To be, or not to be, that is the question" in this way Hamlet asked himself if should be better to act against his enemies or get loose. In the same way we can ask ourselves to "treat (and how) or not treat" the hypertension of the elderly patient. Treatment of hypertension in the elderly is a hotly debated topic because this type of patient has an increased risk of cardiovascular disease, chronic kidney insufficiency and dementia. On the other side it is essential to consider the possible side effects that antihypertensive therapy can cause in the elderly subject: orthostatic hypotension, acceleration of cognitive decline, reduction of coronary perfusion with consequent myocardial ischemia. In the SPRINT trial, in fact, a significant reduction in the risk of cardiovascular events in elderly 
patients is associated with an increase in renal damage and syncope episodes [14,15].

The current guidelines, although advising to treat patients over 65 years who have hypertension, are not aligned on which systolic blood pressure and diastolic blood pressure values a treatment can be started. The members of the US Eighth Joint National Committee recommended in 2014 that in older individuals, antihypertensive treatment should be initiated at SBP values above $150 \mathrm{mmHg}$ and SBP reduced to values lower than $150 \mathrm{mmHg}$ [16]. The 2017 High Blood Pressure Clinical Practice Guideline of the American Heart Association/American College of Cardiology makes a strong recommendation (Level of Evidence A) to initiate antihypertensive drug treatment in individuals of 65 years or older with SBP of 130 mmHg or higher with a treatment goal of less than $130 \mathrm{mmHg}$ [17]. The European Society of Cardiology and the European Society of Hypertension 2018 guidelines recommend that in older patients treated for hypertension, blood pressure should be lowered to $<140 / 80 \mathrm{mmHg}$, but not below $130 \mathrm{mmHg}$ for SBP.

SBP target range of $130-139 \mathrm{mmHg}$ is recommended for people older than 80 years, if tolerated. Blood pressure lowering drug treatment and lifestyle intervention is recommended in fit older patients ( $>65$ years but not $>80$ years) when SBP is in the grade 1 range (140-159 $\mathrm{mmHg}$ ), provided that treatment is well tolerated. In all patients is recommended to initiate an antihypertensive treatment with a two drugs combination, preferably in a single pill combination. The exceptions are frail older patients and those at low risk and with grade 1 hypertension (particularly if SBP is $<150$ $\mathrm{mmHg}$ ). SBP thresholds suggested for initiating treatment are $\geq 140$ mmHg in patients aged $65-79$ and $\geq 160 \mathrm{mmHg}$ in patients aged $\geq 80$; DBP threshold for treatment is $\geq 90 \mathrm{mmHg}$ in both patient groups aged 65-79 and $\geq 80$. The impact of blood pressure-lowering on the well-being of the patient should be closely monitored, because the increased risk of adverse events (e.g. injurious falls) with lower blood pressure values could be more pronounced in older patients in the real-life setting than in the closely monitored conditions of randomized controlled trials. Recommended initial therapy, for all ages, for uncomplicated hypertension, is one pill with dual combination of ACE inhibitor (ACEi)/angiotensin renin blocker $(\mathrm{ARB})+$ calcium channel blocker (CCB)/diuretic. Second step therapy is one pill with a triple combination of ACEi /ARB + CCB + diuretic.

Third step therapy, for resistant hypertension, is 2 pills with triple combination of above + spironolactone or other diuretic, alpha-blocker or beta-blocker. Consider beta-blockers at any treatment step, when there is a specific indication for their use (e.g. heart failure, angina, post-myocardial infarction, atrial fibrillation). Drug initial treatment strategy, for any age, for hypertension and coronary artery disease, is a triple combination of ACEi/ARB + beta-blocker/CCB or CCB + diuretic/beta-blocker or beta-blocker + diuretic. Second step therapy is one pill with a triple combination of above considering initiating therapy when SBP is $130 \mathrm{mmHg}$ in these very high-risk patients with stablished cardiovascular disease. Third step therapy, for resistant hypertension, is 2 pills with triple combination of above + spironolactone or other diuretic, alpha-blocker or beta-blocker. Drug treatment strategy for hypertension and chronic kidney disease, for all ages, is one pill with dual combination of ACEi /ARB + CCB or ACEi /ARB + diuretic (use loop diuretics when eGFR is $<30 \mathrm{~mL} / \mathrm{min} / 1.72 \mathrm{~m}^{2}$ ).

Second step therapy is one pill with a triple combination of ACEi/ARB + CCB + diuretic (use loop diuretics when eGFR is $<30$ $\mathrm{mL} / \mathrm{min} / 1.72 \mathrm{~m}^{2}$ ). Third step therapy, for resistant hypertension, is 2 pills with triple combination of above + spironolactone or other diuretic, alpha-blocker or beta-blocker. A reduction in eGFR and rise in serum creatinine is expected in patients with chronic kidney disease who receive blood pressure therapy especially in those treated with ACEi or ARB [1]. Are there any benefits in treating the elderly patient with antihypertensive therapy and at what values of SBP and DBP is it possible to start a treatment? For many years, advanced age has been a barrier to the treatment of hypertension due to concerns about potential poor tolerability. This approach does not seem appropriate, because evidence from randomized controlled trials has shown that in old and very old patients, antihypertensive treatment substantially reduces cardiovascular morbidity and cardiovascular and all-cause mortality [18]. A recently published meta-analysis by Thomopoulos et al. has identified and analyzed 32 blood pressure-lowering randomized controlled trials with data on 95.549 older patients and 31 randomized controlled trials on 114.009 younger patients. Significant reductions in the relative risk of all major mortality and morbidity outcomes were observed in elderly and young patients without significant differences. Moreover, a reduction of systolic blood pressure between 140 and $150 \mathrm{mmHg}$ in older patients significantly reduces the risk of stroke and major cardiovascular events.

Fatal and nonfatal stroke, fatal and nonfatal coronary heart disease events, fatal and nonfatal hospitalized heart failure, composite of stroke and hospitalized heart failure, cardiovascular death were considered outcomes of mortality and morbidity. Furthermore, no significant differences were found using as a cut-off of 60 or 65 years, and patients treated up to 80 years have shown a significant reduction of stroke, heart failure and major cardiovascular events. The meta-analysis also suggested that it is possible to initiate antihypertensive treatment in individuals over 60 with an untreated baseline systolic blood pressure of 140-149 $\mathrm{mmHg}$ although there are not many clinical trials in elderly patients. This study also shows that the prudent recommendation of not depriving older hypertensive patients with grade 1 systolic blood pressure elevation (systolic 140-159 mmHg and/or diastolic 90/99 $\mathrm{mmHg}$ ) of the possible benefits of treatment is not only founded on experts opinion but is supported by evidence at least when an 
age cut-off of 60 years is used [19]. Regarding the treatment of diastolic hypertension, it is essential to consider the risk of organ hypoperfusion (particularly myocardium) in the elderly patient, who usually has already reduced diastolic blood pressure values.

It is recommended to maintain a diastolic blood pressure target between 70 and $79 \mathrm{mmHg}$, paying attention to the risk of organ hypoperfusion. The results of Thomopoulos' meta-analysis could be compared with the 2008 Blood Pressure Lowering Treatment Trialists Collaboration meta-analysis that did not find significant differences between the effects of BP-lowering treatment in older and younger individuals [20]. It would be useful to create a score that groups frailty syndrome in the elderly to the major patients' comorbidities (renal failure, cognitive impairment, risk of falls and fractures, cardiovascular risk) to identify a score beyond which the introduction of antihypertensive therapy is recommended and useful, as for example, Fried et al have done for the definition of frailty: a clinical syndrome characterized by three or more of the following: unintentional weight loss of $10 \mathrm{lbs}$ or more in the past year, self-reported exhaustion, weakness measured by grip strength, slow walking speed, and low physical activity is a good example [21].

\section{Conclusion}

The treatment of hypertension has been shown to be accompanied by a reduction in mortality and morbidity both in older and younger hypertensive patients. In the elderly hypertensive patients, it should be advised to undertake a hypertensive treatment together with an evaluation of the presence of other comorbidities and the risks associated with multi-therapy, especially in the older. BP should be lowered to a systolic value of 130-139 mmHg and a diastolic value of $<80 \mathrm{mmHg}$ if tolerated. Treated SBP values of $<130 \mathrm{mmHg}$ should be avoided. It is essential to underline that the elderly patient is a difficult patient to treat and therefore deserves a "sartorial therapy" with a careful evaluation of the risks and benefits. Clinicians can successfully respond to the Hamlet question and choice an adequate antihypertensive therapy for older patients. The introduction of a score that groups frailty syndrome in the elderly to the major patients' comorbidities could help to dissolve doubts.

\section{References}

1. Williams B, Mancia G, Spiering W, Agabiti Rosei E, Azizi M, et al. (2018) 2018 ESC/ESH Guidelines for the management of arterial hypertension. J Hypertens 39(33): 3021-3104.

2. Ostchega Y, Dillon CF, Hughes JP, Carroll M, Yoon S (2007) Trends in hypertension prevalence, awareness, treatment, and control in older U.S. adults: data from the National Health and Nutrition Examination Survey 1988 to 2004. J Am Geriatr Soc 55: 1056-1065.

3. Lloyd Jones DM, Evans JC, Levy D (2004) Epidemiology of hypertension in the old-old: data from the community in the 1990s. Am J Hypertens 17(5): 200.

4. Burt VL, Whelton P, Roccella EJ, Brown C, Cutler JA, et al. (1995) Prevalence of hypertension in the US adult population. Results from the
Third National Health and Nutrition Examination Survey, 1988-1991. Hypertension 25(3): 305-313.

5. Thijssen DHJ, Carter SE, Green DJ (2016) Arterial structure and function in vascular ageing : are you as old as your arteries ? 594: 2275-2284.

6. Drayer JIM, Weber MA, Young JL, Wyle FA (1982) Circadian blood pressure patterns in ambulatory hypertensive patients: Effects of age. Am J Med 73(4): 493-499.

7. Caird FI, Andrews GR, Kennedy RD (1973) Effects of posture on blood pressure in the elderly. Br Heart J 35(5): 527-530.

8. Conroy SP, Westendorp RGJ, Witham MD (2018) Hypertension treatment for older people- navigating between Scylla and Charybdis. Age and Ageing 47(4): 505-508.

9. Byyny RL (1990) Hypertension in the elderly. in Hypertension: Pathophysiology, Diagnosis and Management. Raven Press 1869-1887.

10. Kannel WB, Gordon T, Schwarz MJ (1971) Systolic versus diastolic blood pressure and risk of coronary heart disease: The Framingham study. Am J Cardiol 27(4): 335-346.

11. Ettehad D, Emdin CA, Kiran A, Anderson SG, Callender T, et al. (2016) Blood pressure lowering for prevention of cardiovascular disease and death: a systematic review and meta-analysis. Lancet 387(10022): 957967.

12. Lewington S, Clarke R, Qizilbash N, Peto R, Collins R (2002) Age-specific relevance of usual blood pressure to vascular mortality: a meta-analysis of individual data for one million adults in 61 prospective studies. Lancet 360(9349): 1903-1913.

13. Tsai WC, Wu HY, Peng YS, Yang JY, Chen HY, et al. (2017) Association of intensive blood pressure control and kidney disease progression in nondiabetic patients with chronic kidney disease: a systematic review and meta-analysis. JAMA Intern Med 177(6): 792-799.

14. Beddhu S, Chertow GM, Cheung AK, Cushman WC, Rahman M, et al. (2018) Influence of Baseline Diastolic Blood Pressure on Effects of Intensive Compared with Standard Blood Pressure Control. Circulation 137: 134-143.

15. Khan NA, Rabkin SW, Zhao Y, Mc Alister FA, Park JE, et al. (2018) Effect of Lowering Diastolic Pressure in Patients with and Without Cardiovascular Disease. Hypertension 71(5): 840-884.

16. James PA, Oparil S, Carter BL, Cushman WC, Dennison Himmelfarb C (2014) 2014 Evidence-Based Guideline for the Management of High Blood Pressure in Adults Report from the Panel Members Appointed to the Eighth Joint National Committee (JNC 8). JAMA 311(5): 507-520.

17. Whelton PK, Carey RM, Aronow WS, Casey DE, Collins KJ, et al. (2017) 2017 ACC/AHA/AAPA/ABC/ACPM/AGS/APhA/ASH/ASPC/NMA /PCNA Guideline for the Prevention, Detection, Evaluation, and Management of High Blood Pressure in Adults: A Report of the American College of Cardiology/American Heart Association Task Force on Clinical Practice Guidelines. Hypertension 138: e426-e483.

18. Beckett NS, Peters R, Fletcher AE, Staessen JA, Liu L, et al. (2008) Treatment of hypertension in patients 80 years of age or older. N Engl J Med 358: 1887-1898.

19. Thomopoulos C, Parati G, Zanchetti A (2018) Effects of blood pressurelowering treatment on cardiovascular outcomes and mortality: $13^{\wedge}$ benefits and adverse events in older and younger patients with hypertension: overview, meta-analyses and meta regression analyses of randomized trials. J Hypertens 36(8): 1622-1636.

20. Turnbull $F$ (2003) Effects of different blood-pressure-lowering regimens on major cardiovascular events: results of prospectively- designed overviews of randomized trials. Lancet 362(9395): 1527-1535.

21. Fried LP, Tangen CM, Walston J, Newman AB, Hirsch C, et al. (2001) Frailty in older adults: evidence for a phenotype. J Gerontol A Biol Sci Med Sci 56(3): M146-M156. 


\section{ISSN: 2574-1241}

DOI: $10.26717 /$ BJSTR.2019.13.002358

Lorenzo A C. Biomed J Sci \& Tech Res

(C) (i) This work is licensed under Creative

Submission Link: https://biomedres.us/submit-manuscript.php

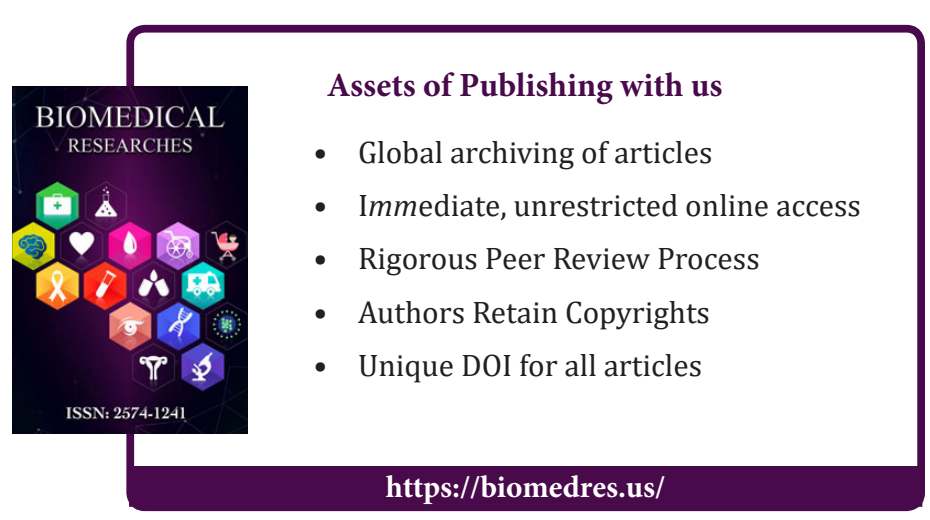

